\title{
La impronta del showrunner en las series de ficción televisiva: El caso de Jenji Kohan
}

\author{
Showrunner-aren aztarna telebistako \\ fikzio-saioetan: Jenji Kohan-en kasua
}

\section{The showrunner's creative imprint on fiction television series: The Jenji Kohan's case}

\section{María José Higueras ${ }^{1}$ \\ Francisco Javier Gómez Pérez²}

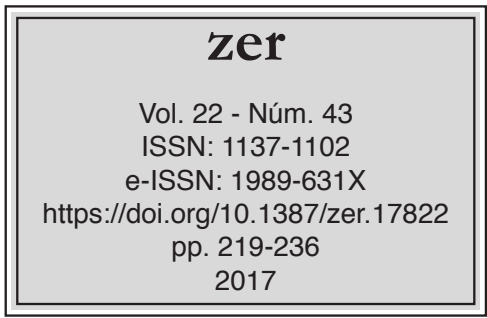

Recibido el 4 de mayo de 2017, aceptado el 21 de septiembre de 2017.

\section{Resumen}

El presente artículo tiene como objetivo analizar la figura de Jenji Kohan como showrunner de la industria televisiva norteamericana, para valorar la existencia de un estilo personal sobre los proyectos que produce. Con dicho propósito, se revisa la biofilmografía de la autora y se evalúa Weeds (2005-2012) y Orange Is The New Black (2013- ) a través de una metodología de análisis que incluye el contexto de producción y los elementos de la narrativa, estética y temática audiovisual. Finalmente, los resultados obtenidos permiten evidenciar una serie de estilemas comunes presentes en ambas series de televisión, conformando el sello creativo de su showrunner.

Palabras clave: Series de televisión; producción; audiovisual; creatividad; showrunner; Jenji Kohan.

\section{Laburpena}

Artikulu honen xedea da Jenji Kohanek Ipar Amerikako telebista-industrian showrunner gisa duen garrantzia aztertzea, ekoizten dituen proiektuetatik haratago estilo pertsonalik ba ote dagoen ikusteko. Xede hori aintzat hartuta, autorearen biofilmografia arakatu dugu, eta Weeds (2005-2012) eta Orange Is The New Black (2013- ) aztertu, ekoizpenaren testuingurua eta

\footnotetext{
Universidad de Granada, mhiguer@ugr.es

2 Universidad de Granada, frangomez@ugr.es
} 
narratibaren, estetikaren eta ikus-entzunezko tematikaren elementuak aintzat hartzen dituen metodologia baten bidez. Lortutako emaitzek agerian uzten dute hainbat ezaugarri berdin ageri direla bi telebista-saioetan. Ezaugarri horiek, hain zuzen ere, showrunner-aren ukitua osatzen dute.

Gako-hitzak: Telesailak; ekoizpena; ikus-entzunezkoa; sormena; showrunner; Jenji Kohan.

\begin{abstract}
The main purpose of this article is taking a look into the personal producer's style of Jenji Kohan as showrunner of the North American television industry. To support this aim, the paper examines the Jenji Kohan's career, and her last audiovisual works -Weeds (2005-2012) and Orange Is The New Black (2013- )- thanks to the analysis of context production, and narrative, aesthetic and thematic aspects. Finally, we discuss the findings obtained in order to elucidate the common labels which constitute the showrunner's creative hallmark and reveal her creative responsibilities.
\end{abstract}

Keywords: TV series; production; audiovisual; creativity; showrunner; Jenji Kohan. 


\section{Introducción}

El productor audiovisual constituye un perfil profesional que, a pesar de la limitada atención recibida en comparación con otros profesionales del medio, desarrolla una serie de funciones y responsabilidades que conllevan una intervención esencial en el proceso de creación de la obra, tanto en el contexto económico como en el creativo (Fernández-Díez \& Martínez-Abadía, 1994, 2010; Zettl, 1996; Barroso, 2002; Jacoste, 2004; Millerson, 2009). Por lo tanto, el productor ejecutivo "es central en cada aspecto del proyecto, desde la chispa de una idea hasta la pieza de trabajo tangible" (Kellison, 2009: 1) ${ }^{3}$.

$\mathrm{El}$ reconocimiento del productor como ente creativo es especialmente notable en la industria televisiva a partir de los años ochenta del siglo XX, cuando ésta es conocida como The Producer's Medium (Newcomb \& Alley, 1983: 8):

El productor es la persona a cargo de la producción televisiva, quien establece el espíritu de trabajo y dicta los estándares que se deben cumplir. Idealmente, como jefe del equipo creativo, el productor es a la vez empresario y artista, se preocupa por la administración y los detalles presupuestarios mientras nutre el talento y proporciona la visión del proyecto ${ }^{4}$.

Dichas consideraciones adquieren una atención significativa en el siglo XXI, dentro del marco de la reputación que ha logrado esta figura en las series de ficción contemporáneas durante la Tercera Edad Dorada de la Televisión, caracterizada por la evolución del drama televisivo gracias a los canales de cable (Buonanno, 2008; Kallas, 2014). Es entonces cuando, a pesar de haber surgido en los años noventa (Newman \& Levine, 2012), se generaliza el término de showrunner para designar al "responsable de la dirección creativa del programa" (Cascajosa, 2016a: 23), que desarrolla el máximo nivel de liderazgo al intervenir en la supervisión de todas las fases del proceso. Por lo tanto, el showrunner está capacitado, entre otras cuestiones, para controlar la financiación, negociar con los ejecutivos de la cadena, contratar al equipo técnico y artístico, y revisar los guiones (Gitlin, 2000; Wirth \& Melvoin, 2004; Douglas, 2011; Gervich, 2008; Bennett, 2014; Landau, 2014; De Rosendo \& Gatell, 2015; Cascajosa, 2016a).

Dichas funciones suponen una marca creativa sobre el producto, advertida por la audiencia al reconocer un estilo cinematográfico concreto (Bordwell \& Thompson, 2012) que, entre otras vertientes, afecta a la narrativa, a la estética y a la temática. Se trata de establecer lo que Kubey (2009: 122) denomina esquema del proyecto:

\footnotetext{
3 En el texto original: "The producer is central to every aspect of a project- from the wisp of an idea to a tangible piece of work" (Kellison, 2009: 1). Traducción propia en el artículo.

${ }^{4}$ En el texto original: "The producer is the person in charge of a TV production, who establishes the working spirit and dictates the standards to be met. Ideally, as head of the creative team; the producer is both businessman and artist, caring about administration and budgetary details while nurturing the talent and providing the vision for the project" (Newcomb \& Alley, 1983: 8). Traducción propia en el artículo.
} 
"estilo, tono, temática y personajes"5; y Cascajosa (2007), universo de ficción, el cual es el sello del autor. A través de dichos elementos podemos reconocer lo que se conoce como su voz (Kirkpatrick, 2015; De Rosendo \& Gatell, 2015; Bennett, 2014), esto es, la impronta personal del showrunner, que "definen su trabajo como el de un autor, y no como un simple productor ejecutivo" (Raya, 2012: 408).

En este contexto resulta pertinente considerar la noción de autoría en el medio audiovisual, cuyo origen se halla en la politique des auteurs, desarrollada por los críticos franceses a través de la revista Cahiers du Cinéma y posteriormente aplicada a la industria americana a través de the auteur theory. Dichas corrientes asignan al director de la película la capacidad de aplicar una firma personal a través de ciertas características recurrentes (Sarris, 2004). Sin embargo, "el concepto de autoría en televisión se halla intrínsecamente unido al showrunner" (Steiner, 2015: 183) ${ }^{6}$, que, por otra parte, debe alcanzar el equilibrio entre la voz individual y el proceso colaborativo que supone la creación de una serie de ficción televisiva (Martin, 2013).

\section{Objetivos y metodología}

El presente artículo pretende conocer los estilemas hallados en las series de ficción televisiva de un mismo showrunner y, de este modo, verificar la hipótesis que considera a dicho profesional autor de los proyectos que produce y, de este modo, poseedor de un estilo creativo que aplica a los mismos. Además, a través de este objetivo se busca dilucidar y poner en valor las implicaciones y competencias artísticas que este productor lleva a cabo dentro de la industria televisiva.

Con el fin de optimizar el desarrollo del estudio, se ha realizado una revisión bibliográfica que permite enmarcar el concepto que tratamos. Por una parte, consideramos la investigación a través de las series de televisión americanas que Cascajosa (2005, 2006, 2007, 2016a, 2016b) ofrece en su bibliografía. Además, debemos destacar los textos dedicados a los procesos de producción televisiva hallados en los trabajos de García de Castro (2002), Clements (2004), Diego (2005, 2010), Gervich (2008), Kellison (2009), y Douglas $(2011,2015)$.También hemos prestado atención a las densas entrevistas a creadores de series de ficción recogidas por Longworth (2000, 2002), Priggé (2005), Kubey (2009) y Kallas (2014); y a la aplicación de la teoría del autor al medio televisivo expuesta por Newcomb y Alley (1983) e incisivamente estudiada por Perren y Schatz (2015).

Por otra parte, se toman como referencia distintos análisis de series de ficción televisiva de índole y objetivos variados. Al respecto, destacamos el trabajo sobre productos norteamericanos llevado a cabo por Tous-Rovirosa $(2009$, 2010), el análisis del lenguaje de las series de ficción realizado por García-Fanlo (2016), el estudio del creador de ficción televisiva, Joss Whedon, a través de sus series más representativas (Raya, 2012) o la presentación de diferentes series en función de sus características más notables (Thompson \& Mittell, 2013).

5 En el texto original: "the style, tone, subject, theme and characters" (Kubey, 2009: 122). Traducción propia en el artículo.

6 En el texto original: "Nowadays, authorship in television is often intrinsically tied to the showrunner (...)” (Steiner, 2015: 183). Traducción propia en el artículo. 
Tomando este marco teórico como referencia, se lleva a cabo la aproximación al caso concreto de Jenji Kohan, profesional de la ficción televisiva norteamericana, y a sus últimas producciones: Weeds (Showtime: 2005-2012) y Orange Is The New Black (Netflix: 2013- ) -en adelante OITNB-. El desarrollo del estudio implica estructurar el artículo en dos partes. En primer lugar, se expone una breve revisión biofilmográfica de la autora con el objetivo de comprender y contextualizar las obras analizadas. En este punto, se atiende al contexto y modos de producción imperantes en el periodo actual, conocido como Tercera Edad Dorada de la Televisión (PérezGómez, 2011), donde la figura del showrunner adquiere un notable reconocimiento y relevancia dentro y fuera del medio televisivo (Newman \& Levine, 2012).

A continuación, la investigación profundiza en los productos audiovisuales anteriormente señalados, que son visualizados desde una perspectiva crítico-analítica y, posteriormente, examinados y comparados con el fin de dilucidar los rasgos compartidos por ambas series. Para ello, la metodología de análisis aplicada toma como referencia la guía propuesta por Sánchez-Noriega en Historia del cine (2006: 61-66), adaptándola a los objetivos señalados en el presente estudio. El método incluye las siguientes cuestiones: 1) contexto de producción y ficha técnico-artística, para cuya configuración se tendrán en cuenta las anotaciones de Jenji Kohan en medios de comunicación de repercusión internacional, así como la información facilitada en la base de datos especializada en productos audiovisuales -Internet Movie Database-IMDb-; 2) sinopsis, abordada desde el visionado del producto; 3 ) elementos formales del texto fílmico, esto es, códigos visuales, sonoros y sintácticos que son analizados en función de la narrativa y el lenguaje audiovisual (Edgard-Hunt, Marland \& Rawle, 2010); 4) elementos formales del relato, que incluyen el punto de vista, tiempo, estructura narrativa, personajes y espacios, para cuyo desarrollo se atiende a los textos de Fernández-Díez y Martínez-Abadía (2003), Gordillo-Álvarez (2009) y Carrasco (2010), entre otros; 5) temática, considerando la influencia de la libertad creativa permitida en los canales de cable (Perren, 2011; Douglas, 2015); y 6) recepción, en función de la información aportada por la base de datos señalada.

De forma genérica, las series de ficción televisiva son evaluadas atendiendo a dichos parámetros para extraer sus características más notables, prestando especial atención a las implicaciones de Kohan durante el proceso de producción y creación, así como a los elementos narrativos, estéticos y temáticos comunes que conformarían la impronta personal de la creadora.

\section{Revisión biofilmográfica de Jenji Kohan}

Con el objetivo de comprender las funciones, responsabilidades y competencias desarrolladas por Jenji Kohan en el contexto de creación de los productos analizados, debemos considerar el origen del perfil del showrunner en la industria de las series de ficción televisiva contemporáneas. En este sentido, el modelo de writer-producer, antecedente del showrunner, está presente en el proceso de producción de las primeras series televisivas, como en la comedia I Love Lucy (Jess Oppenheimer, CBS: 1951-1957) (Banks, 2013) y, posteriormente, en las producciones de MTM Enterprises, destacadas por la libertad creativa y el reconocimiento asignado al guionista (Feuer, 1984; Gitlin, 2000; Pearson, 2005), lo que permitió a Steven Bochco aplicar 
un estilo personal a la paradigmática serie Hill Street Blues (NBC: 1981-1987) (Longworth, 2000).

Sin embargo, en el siglo XXI se inicia una época marcada por una serie de factores históricos, culturales y tecnológicos que motivan el uso generalizado del término showrunner -título no oficial que responde al crédito de "productor ejecutivo" (Gervich, 2008)- y, con ello, su reconocimiento y popularidad. En esta línea, consideramos el liderazgo de los canales de cable (HBO, Showtime, CNN y AMC) y sus posibilidades creativas -historias complejas, personajes amorales y temas controvertidos (Tous-Rovirosa, 2009; Martin, 2013; Douglas, 2015)- como punto de partida de la nueva era, popularmente establecido con la serie creada y producida por David Chase, The Sopranos (HBO: 1999-2007) (Sepinwall, 2012).

Además, las series de ficción contemporáneas, entre las que podemos destacar Game of Thrones (David Benioff \& D. B. Weiss, HBO: 2011- ), Girls (Lena Dunham, HBO: 2012-2017), The Walking Dead (Frank Darabont, AMC: 2010- ) o Sons of Anarchy (Kurt Sutter, FX: 2008- 2014) (Sepinwall, 2012), son en su mayoría creadas y producidas por un profesional del audiovisual dedicado plenamente al medio televisivo (Martin, 2013) cuyas competencias y responsabilidades se aplican en todas las fases y departamentos del proceso de producción. En esta línea, Newman y Levine (2012) indican que "en la era de la convergencia, el showrunner realiza tanto tareas de escritura como otras, integrando claramente diferentes competencias y preocupaciones". (39) ${ }^{7}$.

Finalmente, indicamos una serie de factores asociadas al desarrollo de las nuevas tecnologías que también han contribuido a la circunstancia estudiada. Se trata de las oportunidades que ofrece Internet a las plataformas de video bajo demanda, destacando el caso de Netflix (Douglas, 2015); y como lugar de encuentro y discusión entre los showrunners y la audiencia, gracias a las redes sociales (Perren \& Schatz, 2015; Bennett, 2014; Cascajosa, 2016b).

En relación al presente trabajo, la elección de la figura de Kohan y su producción televisiva como objeto de estudio y análisis se halla justificada por diversas razones. Por una parte, estamos ante uno de los referentes femeninos más destacados y representativos del panorama de la producción audiovisual de series de ficción, cuya biografía demuestra un amplio bagaje en el contexto que estudiamos. Además, resulta especialmente interesante que desarrolle su labor dentro de la industria norteamericana, donde el concepto de showrunner se halla plenamente establecido. Por otra parte, el perfil de Kohan aúna a la perfección las cualidades que definen a este tipo de productor como la persona que desempeña labores de gestión administrativa, toma decisiones creativas, posee la idea y se implica activamente en la escritura del guion (Raya, 2012).

Jenji Leslie Kohan -California (Estados Unidos), 1969- inició su carrera como guionista en Will \& Grace (David Kohan \& Max Mutchnick, NBC: 1998-2006), puesto que también desempeñó en El príncipe de Bel-Air (The Fresh Prince of BelAir, Andy Borowitz \& Susan Borowitz, NBC: 1990-1996), Sexo en Nueva York (Sex and The City, Darren Star, HBO: 1998-2004) o Las chicas Gilmore (Gilmore Girls, Amy Sherman-Palladino, WB: 2000-2007).

\footnotetext{
En el texto original: "the convergence-era showrunner is active in both writing and non-writing tasks on an ongoing basis, integrating two distinctly different sets of skills and concerns" (Newman y Levine, 2012: 39). Traducción propia en el artículo.
} 
En la mayoría de estas obras desempeña ciertas labores de producción, aunque el doble perfil no queda configurado hasta su trabajo en The Stones (David Kohan, Max Mutchnick \& Jenji Kohan, CBS: 2004). No obstante, la temprana cancelación del proyecto lleva a considerar Weeds como su ópera prima, es decir, la primera serie de televisión donde ejerce plenamente como creadora, escritora y productora; funciones que igualmente desarrolló en $O I T N B$.

\section{Análisis de las producciones de Jenji Kohan}

A continuación, se expondrán los resultados generales derivados del análisis de las series de ficción Weeds y OITNB según el modelo y parámetros anteriormente indicados. Las semejanzas encontradas nos permitirán valorar el alcance de la labor del showrunner en el terreno creativo de la producción, así como su marca personal sobre dichas obras audiovisuales.

\subsection{Weeds}

Con la finalidad de estudiar el contexto de producción de Weeds, debemos considerar la motivación de Kohan hacia un proyecto innovador alejado de lo convencional. Por ello la trama gira en torno a una antiheroína y una droga que, a pesar de estar prohibida en Los Ángeles, es consumida de forma común. "Creo que lo que hizo que la serie fuese tan efectiva cuando aterrizó es que estábamos viviendo un periodo muy conservador en el país" ", afirma su creadora en una entrevista para Anatomy of a Script (2014).

La showrunner, alejándose de la concepción de la vida idealizada predominante en la programación de las networks, busca abandonar los extremos. Por esta razón, según cuenta en el programa de radio "Fresh Air" (2008), en el 2005 acude con su proyecto y cinco palabras - "Suburbano, viuda, marihuana, negocio, mama"9- a Showtime, caracterizada por aceptar temas controvertidos y no limitar la creatividad del autor (Cascajosa, 2005; Valderrama, Boo \& Costas, 2013). Así, la cadena financió dicha serie durante sus ocho temporadas.

En cuanto al argumento, Weeds propone una historia que, en el momento de su estreno, resultaba notablemente novedosa: una madre de familia se queda repentinamente viuda y decide vender marihuana para mantener a sus hijos. Se presenta así una vuelta de tuerca sobre los convencionalismos, una ácida crítica a la sociedad que impone un estilo de vida perfecto, a la vez que oculta tras las apariencias a personas envidiosas y superficiales. Nancy Botwin -Mary-Louise Parker-, la protagonista, rompe con todas las normas y, sin parecer importarle las consecuencias, adquiere un estilo de vida alejado de lo políticamente correcto, justificando sus actos por razones familiares. Dicha trama resulta notablemente

\footnotetext{
8 En el vídeo original del evento Anatomy of a Script (Schiff, R. \& Holzman, W., 2014) de la Writers Guild Fundation: "I think that what made the show so effective when it landed is that it came at a time when we were living in a sort of very conservative period in the country". Traducción propia en el artículo.

9 En el audio original del programa "Fresh Air" (Terry Gross \& Danny Miller: 2008): "Suburban widowed pot-dealing mom". Traducción propia en el artículo.
} 
similar a la posteriormente presentada por Vince Gilligan en Breaking Bad (AMC: 2008-2013), lo que le supuso ciertos inconvenientes en el proceso de venta del producto (Martin, 2013).

Acudiendo a los elementos formales del texto debemos indagar en los códigos visuales, sonoros y sintácticos que se dan en el conjunto de la serie. Se trata de rasgos que han de ser supervisados por el showrunner para asegurar la continuidad de un producto cuya serialidad permite y propicia que no todos los episodios sean dirigidos por la misma persona (Kallas, 2014). En Weeds prevalecen planos sencillos -plano general, plano entero, y plano medio-, entre los que son destacados otros más complejos, como los planos cenitales en momentos conflictivos y, especialmente, en el final de varios capítulos. Respecto a la iluminación y colorimetría, observamos escenas muy claras complementadas con tonalidades amarillentas y anaranjadas. Es frecuente, en este sentido, hallar planos predominantemente grises, donde algún elemento de color verde destaca sobre el resto.

En cuanto a los códigos sonoros, la música contiene interesantes connotaciones significativas cuya inclusión responde a razones de índole narrativo. Los diálogos, por su parte, son utilizados como herramienta de crítica a través del uso de metáforas e ironías. En este sentido, es esencial visionar la serie en versión original para poder apreciar los acentos de personajes norteamericanos y mexicanos.

Finalmente, el producto presenta un ritmo ágil y rápido gracias a una serie de técnicas como el montaje al corte y la ausencia de fundidos, así como la presencia de ciertas secuencias de montaje, que aglutinan planos de manera no convencional planos repetidos, planos a cámara rápida, planos hacia delante y hacia atrás, o planos cuyo audio no corresponde con la imagen-.

Analizando los elementos formales del relato, hallamos que la serie se estructura en ocho temporadas compuestas por entre diez y trece capítulos -esquema frecuente en las series emitidas por canales de cable (Valderrama et al., 2013) - de aproximadamente media hora de duración.

Weeds responde a un modelo serial con subtramas episódicas lo que, en palabras de Gordillo-Álvarez (2009: 97), consiste en:

Un producto televisivo basado en la serialidad, con tramas que se enredan y se alargan durante numerosos capítulos del programa (...) Sin embargo, cada episodio acostumbra a configurarse a partir de una cuestión esencial que se resuelve dentro del capítulo.

Por otra parte, estamos ante una dramedy o dramedia, formato que aúna matices del drama y la comedia para provocar el humor a través de situaciones trágicas que llegan a ser absurdas. Este tipo de serie presenta espacios exteriores e interiores, repartos corales y una estructura terciaria que incluye varias tramas (Carrasco, 2010: 184-193). Además, se caracteriza por una visualidad cinematográfica gracias a las tipologías de planos y movimientos de cámara que suponen una notable calidad estética (Gómez-Martínez, 2015). Sin embargo, es esencial advertir que, a diferencia de la mayoría de este tipo de productos, el que aquí estudiamos presenta una duración 
propia de la sitcom -comedia de situación-, esto es, 25 minutos aproximadamente (Gordillo-Álvarez, 2009; Carrasco, 2010).

En cuanto al punto de vista, la ficción cuenta con una focalización externa, la técnica más empleada en el audiovisual, que consiste en ir variando la perspectiva de la cámara de manera que el espectador pueda observar lo que acontece en la escena desde una visión cambiante y externa a la acción en sí.

El tiempo cinematográfico, por su parte, es lineal y sin flashbacks niflashforwards reiterantes: podemos apreciar un salto al pasado para mostrar la muerte del padre de la familia, y únicamente hay dos grandes elipsis que tienen lugar durante el periodo en el que la protagonista ingresa en prisión y justo antes de finalizar la última temporada. Además, atendiendo a las edades de los hijos de Nancy, deducimos que la trama se desarrolla aproximadamente durante catorce años.

En cuanto a la localización, es cambiante: desde la ciudad ficticia de Agrestic, a diversas zonas de Estados Unidos, México y Europa. Además, la mayoría de las tramas se desarrollan en interiores, aunque también asistimos a relevantes acontecimientos que tienen lugar en las calles y carreteras, especialmente durante el periodo que transcurre en una caravana.

Por otra parte, los personajes responden a un modelo alejado de los estereotipos y se asemejan a figuras reales, atendiendo al objetivo de Kohan de romper con lo convencional y buscar la identificación por parte de la audiencia (Anatomy of a Script, 2014). Se trata de individuos diferentes, conflictivos y problemáticos, que se relacionarán con otras personas y espacios más amables, dando lugar a situaciones en las que se puede entrever una clara contraposición entre lo legal y lo prohibido.

Nancy Botwin podría definirse como una madre coraje, sin encajar plenamente en dicho canon. Se presenta como una persona fuerte que, lejos de lamentarse por la muerte de su marido, se introduce en el negocio de la marihuana para hacer frente a su precaria situación económica. En este proyecto la protagonista demuestra ser hábil, fría y resuelta, pues, sin olvidar los irrisorios obstáculos que atraviesa, experimenta auténticos momentos de suerte y éxito.

Hallamos a una mujer ambiciosa y competitiva que está totalmente enganchada al tráfico de drogas, sin importarle los riesgos y peligros que ello supone, de los que parece no ser totalmente consciente. Nancy es una auténtica antiheroína que, aún sin actuar de manera socialmente correcta, hace que nos sintamos cómodos o, incluso, identificados con ella. De este modo, el tratamiento que recibe el género femenino en Showtime -United States of Tara (Diablo Cody, 2009-2011), The L Word (Michele Abbott, Ilene Chaiken \& Kathy Greenberg, 2004-2009) - contribuye a que sea considerado:

Como un ejemplo de fórmula en la que las mujeres fuertes, imperfectas, próximas al vicio y a la perversión, han destacado sobre un hombre que no reacciona en una sociedad donde lo femenino en el audiovisual se abre puertas antes inexploradas (Valderrama et al., 2013: 44).

Nancy es acompañada por sus hijos Shane -Alexander Gould- y Silas -Hunter Parrish-, cuyas vidas se verán afectadas por el narcotráfico. Además, la familia es 
completada por su cuñado, Andy Botwin -Justin Kirk-, que trabaja para ella junto con otros vecinos: Doug Wilson -Kevin Nealon- y Dean Hodes -Andy Milder-.

El reparto es completado con un extenso abanico de personajes secundarios de diferente raza, nacionalidad o clase social, que irán entrando y saliendo de las tramas y, con ello, de la vida personal y profesional de Nancy.

En cuanto a la temática de la serie, se abordan tanto los temas de contenido como los mensajes generales que se pueden extraer del visionado de la obra (SánchezNoriega, 2006). En primer lugar, Weeds propone una reflexión acerca de los valores sociales y morales, transformándolos de manera que, aun rayando la ilegalidad, podrían llegar a ser justificados por el bienestar familiar. No obstante, este fin acaba abandonándose al entrar en juego la avaricia, la ambición y la violencia. Se trata de factores negativos que suelen presentarse de manera triunfadora. La narración concluye, sin embargo, con un regreso al inicio, a la necesidad de mantener a la familia unida para alcanzar la estabilidad y la felicidad.

Por otra parte, en el desarrollo de la serie podemos destacar un conjunto de elementos que van guiando el trascurso de la misma. Estamos ante contenidos tabú que conforman la innovadora temática que comienza a predominar en las ficciones de comienzo del siglo XXI: el elevado componente sexual -homosexual y transexual-, la contraposición entre la ley y la droga, o el patente choque cultural que supone la existencia de personajes de diferente nacionalidad, raza y religión (Perren, 2011; Nahum, 2014; Cascajosa, 2016b). Dichos elementos son patentes en otros productos de la época como Californication (Tom Kapinos, Showtime: 2007-2014), American Horror Story (Ryan Murphy, FX: 2011- ) o Sense 8 (J. Michael Straczynski, Lana Wachowski \& Lilly Wachowski, Netflix: 2015-2017).

Finalmente, debemos señalar que Weeds ha obtenido la aceptación de la crítica, como demuestran numerosos premios y nominaciones. Destacamos el Globo de Oro a la Mejor Interpretación Femenina de Comedia para Mary-Louise Parker (2006), así como la nominación a Mejor Serie de Televisión (2006, 2007 y 2009); y el Premio Emmy a la Mejor Fotografía para Serie de Media Hora (2010) y a la Mejor Mezcla de Sonido (2009) (IMDb: Weeds, 2017).

\subsection{Orange Is the New Black}

La siguiente serie de televisión está basada en el libro autobiográfico de Piper Kerman, donde relata su temporada en prisión. Según un artículo en la versión digital del periódico Excelsior fueron dos características de la novela lo que animaron a Kohan a adaptar el texto a la televisión: trata sobre personas muy atrayentes y dispares, y las reúne en un espacio cerrado (Ávila Morales, 2013).

Sin embargo, para vender todos estos personajes poco amables se precisaba de un "caballo de Troya"10, atendiendo a las palabras de Kohan en el programa radiofónico "Fresh Air" (2013). Por lo tanto, una joven blanca americana será el punto de partida para atraer a la audiencia y poder mostrarle historias más complejas.

Por otra parte, Netflix se presenta como la opción más adecuada para producir el proyecto porque permite a Kohan una gran libertad creativa. En este sentido, la

\footnotetext{
${ }^{10}$ En el audio original del programa "Fresh Air" (Terry Gross \& Danny Miller: 2013): "Piper was my Trojan Horse". Traducción propia en el artículo.
} 
autora añade en la citada entrevista de radio que "lo mejor de Netflix fue que llevó los 13 episodios a la vez, sin un piloto. Fue un milagro. El sueño de todo showrunner, sólo centrarse en la serie y poner fe en tu trabajo" "11. Además, la plataforma de video bajo demanda permite consumir sus productos cuándo y dónde el espectador quiera, así como darle acceso a las temporadas completas (Douglas, 2015).

En cuanto al argumento de esta ficción, Piper Chapman -Taylor Shilling- es una chica guapa e inteligente que procede de una familia acomodada y está comprometida con su novio, Larry Bloom -Jason Biggs-. Sin embargo, esta idílica situación se ve alterada cuando le notifican que se ha reabierto un caso de tráfico de drogas ocurrido hace diez años en el cual Piper se involucró animada por su entonces novia, Alex Vause -Laura Prepon-. Así, decide entrar voluntariamente a la cárcel de mínima seguridad para mujeres de Litchfield con la intención de evitar ser juzgada.

La trama principal avanza gracias a otras secundarias que, por una parte, nos muestran los conflictos del resto de personajes dentro de la cárcel y, por otra, dan a conocer su vida anterior. Estos flashbacks suponen un interesante resultado narrativo al intercalar las escenas independientemente del factor temporal, así como por el contraste estético a nivel de espacio, luz y color. Dichos saltos temporales encuentran su máxima expresión en la ficción televisiva contemporánea Lost (J.J. Abrams, ABC, 2004-2010) (Tous-Rovirosa, 2009; Sepinwall, 2015) que "en producción, transgredió las formas tradicionales de narrar la ficción televisiva recurriendo a una compleja desfragmentación tanto espacial como temporal del relato" (García-Fanlo, 2016: 82).

Respecto a los códigos visuales, predomina una realización básica -planos generales y panorámicos, junto con otros más cerrados- y una iluminación plana y uniforme. La colorimetría, por su parte, es presidida por el caqui de los uniformes de las presas, favoreciendo un ambiente gris y homogéneo en el que suele destacar algún elemento de color rojo o naranja.

Por otra parte, los diálogos se convierten en una herramienta esencial para, de manera subliminal, atacar al sistema carcelario. Encontramos interesantes juegos de palabras que únicamente podemos apreciar en la versión original, la cual también es recomendable si queremos advertir las expresiones en español de los personajes latinos. Además, las canciones están cargadas de significado, tanto por la forma de presentarlas como por su propio contenido.

En cuanto al ritmo, debemos apreciar que el montaje no incluye ningún fundido a negro, lo que propicia que la serie sea ágil de consumir, permitiendo visionar un capítulo tras otro -gracias a las opciones que proporciona la plataforma Netflix-sin experimentar una ruptura patente entre ellos.

La producción cuenta, hasta el momento, con cuatro temporadas de trece episodios cada una, cuya duración es aproximadamente de 50 minutos. La vida de Piper se plantea como trama principal, aunque las subtramas creadas por Kohan van tomando relevancia: las que hacen referencia al pasado de las presas suelen autoconcluir en el mismo episodio, y las que protagonizan en tiempo presente abarcan varios capítulos.

\footnotetext{
${ }^{11}$ En el audio original del programa "Fresh Air" (Terry Gross \& Danny Miller: 2013): "The greatest thing about going to Netflix was that I pitched it in the room, and they ordered 13 episodes without a pilot. That's miraculous. That is every showrunner's dream, to just 'go to series' and have that faith put in your work". Traducción propia en el artículo.
} 
Conforme avanza la serie, la historia de Piper se equipara, e incluso queda en un segundo plano, respecto a las protagonizadas por otros personajes.

Estos parámetros hacen que nuevamente hablemos de un modelo serial con subtramas episódicas (Gordillo-Álvarez, 2009) que responde al formato de dramedy o dramedia (Carrasco, 2010), donde destacamos un tono realista que permite presentar a personajes complejos que evolucionan con el transcurso de las tramas (GómezMartínez, 2015).Estamos, por lo tanto, ante repartos corales, localizaciones variadas, estilo cinematográfico y situaciones tragicómicas que constituyen una narración, cuya duración se ajusta a los requisitos indicados por dichos autores, esto es, entre 45 y 50 minutos.

La focalización, por su parte, es externa, lo que permite presenciar las acciones desde diferentes puntos de vista, cambiando la perspectiva en función de los personajes que protagonicen las tramas.

Respecto al tiempo, son especialmente importantes los flashbacks que nos muestran la vida de las presas antes de ser encarceladas. A través de estas escenas podemos ver cómo eran dichas mujeres en el mundo exterior, quedando patente que desarrollan una personalidad diferente para sobrevivir dentro de la cárcel, ideas que Kohan señaló en una entrevista para la web de cine y televisión IndieWire (Willmore, 2013).

Además, entendemos que la serie se desarrolla durante los quince meses a los que Piper Kerman fue inicialmente condenada a prisión. Sin embargo, Kohan estira dicho periodo gracias a la ficción que complementa la historia, por lo que a partir de la tercera temporada podemos advertir que este tiempo es sobrepasado.

Las localizaciones predominantes se hallan dentro del recinto carcelario de Litchfield (Estados Unidos), aunque también podemos destacar los exteriores hallados en los flashbacks y en el mundo paralelo de la protagonista en tiempo presente.

Respecto a los personajes, el reparto es presidido por Piper Chapman -Taylor Shilling-, la chica de clase media-alta que representa el lado amable de la sociedad y desarrolla un perfil en el que cualquier espectador podría sentirse identificado. Sin embargo, a través de sus errores se pone de manifiesto que la imperfección humana no depende de los estamentos sociales. Por otro lado, aunque no deja de mostrar cierta debilidad, advertimos una evolución en la actitud de la protagonista, desde la armonía con la que entra en la cárcel, hasta la última temporada, cuando lidera alguna de las tramas más violentas de la narración.

Como ya hemos señalado, estamos ante un nuevo papel desarrollado por el género femenino en las series de ficción norteamericana contemporáneas, que podemos encontrar independientemente del canal de emisión: tanto en dramas -Revenge (Mike Kelley, ABC: 2011-2015), Big Little Lies (David E. Kelley, HBO: 2017) o The Handmaid's Tale (Bruce Miller, Hulu: 2017- )-, como en comedias -2 Broke Girls (Whitney Cummings \& Michael Patrick King, CBS: 2011- ) o Mom (Gemma Baker, Eddie Gorodetsky \& Chuck Lorre, CBS:2013- )- .

El resto del casting es completado por un grupo de mujeres muy diversas en su edad, inquietudes, raza, nacionalidad y religión, que hacen posible un esquema narrativo caracterizado por el desarrollo de tramas violentas y conflictivas, a la vez que irrisorias e ilógicas.

Galina Reznikov o Red -Kate Mulgrew- es una cocinera de origen ruso que, desde un puesto de autoridad, recibe el respeto y temor del resto de las presas. Sin 
embargo, también es considerada una madre por Nicky Nichols -Natasha Lyonne-, caracterizada por sus problemas de drogodependencia; y Lorna Morello - Yael Stone-, que oculta sus problemas psicológicos con simpatía y amabilidad.

En cuanto a los personajes afroamericanos, Tasha Jefferson o Taystee -Danielle Brooks- y Poussey Washington -Samira Wiley- representan la amistad indestructible que se suele crear en este tipo de espacios. Sin embargo, la figura negra más interesante es la de Suzanne Warren o Crazy Eyes -Uzo Abuda-, cuya inestabilidad emocional protagonizará algunas de las tramas más importantes del discurso.

Por otra parte, respecto al grupo formado por las chicas latinas, resulta interesante hacer referencia a la relación entre Dayanara Díaz -Dascha Polanco- y su madre Aleida Díaz -Elizabeth Rodríguez-, en la que, a pesar de sus diferencias, encuentra un gran apoyo al quedar embarazada.

El elenco es completado, entre otras, por Pennsatucky -Taryn Manning-, cuya obsesión religiosa choca con su carácter conflictivo; Sophia Burset -Laverne Cox-, que deberá enfrentarse a los problemas derivados de su transexualidad; y la revolucionaria monja Jane Ingalls -Beth Fowler-.

Por otra parte, respecto a los trabajadores de la cárcel, hallamos perfiles incompetentes que destacan por los abusos de George Méndez o Pornstache -Pablo Schreiber-; la falta de imposición de Joe Caputo -Nick Sandow-; y la empatía de Sam Healy -Michael Harney-.

En cuanto a los aspectos temáticos, OITNB nos presenta un claro contraste entre la libertad y la opresión -el exterior y el interior de la cárcel-. En esta contraposición de dos mundos podemos comparar la elegancia, el dinero y las comodidades del primero; con la injusticia, la corrupción y el conflicto del segundo. Sin embargo, poco a poco la vida fuera de la prisión deja de ser relevante para dar paso a la que ocurre dentro de ésta, que se convierte en la verdadera realidad de los personajes.

En este contexto, los sentimientos y las emociones se hacen patentes a diferentes niveles. Por una parte, la amistad, el amor y el sexo -especialmente entre mujeres-, están muy presentes durante toda la narración. Sin embargo, factores negativos como la frustración, la violencia o la locura, protagonizan la mayoría de las tramas que se desarrollan durante el transcurso de la serie.

Finalmente, advertimos una positiva recepción que, entre otros factores, se hace patente a través del Globo de Oro a Mejor Serie de Televisión (2015) y el Premio Emmy a Mejor Interpretación Femenina de Reparto en Serie de Comedia a Uzo Aduba (2014), a Mejor Casting en Serie de Comedia (2014) y a Mejor Edición Monocámara (2014) (IMDb, Orange is the New Black: 2017).

\section{Conclusiones}

Tras este análisis de los trabajos creados por Kohan podemos extraer un conjunto de elementos que configuran sus producciones, otorgándoles una entidad narrativa, estética y temática. Atendiendo a estos factores podemos llegar a una serie de conclusiones que permiten discutir y responder a los objetivos propuestos.

En primer lugar, se advierte que, a pesar de que Weeds es una historia original y $O I T N B$ está inspirada en un libro autobiográfico, estamos ante dos proyectos novedosos y controvertidos, que fueron producidos y emitidos en canales que apuestan 
por la innovación formal y temática, abogando por la creatividad y las nuevas formas de consumo audiovisual.

Por otra parte, resulta destacable que en ambos casos a Kohan se le asigna el título de guionista y de productor ejecutivo en las fichas técnicas correspondientes. Además, su nombre también aparece tras "created by" al final de los títulos de inicio. De esta forma, considerando las referencias al término de showrunner, podemos apreciar que Kohan desarrolla este perfil al desempeñar dichos puestos de la industria audiovisual.

En cuanto a la sinopsis argumental, estamos ante historias que, aunque a priori parecen muy diferentes, presentan una evolución de las circunstancias muy similar. Mayores semejanzas encontramos si atendemos a los elementos formales del texto que conforman la experiencia estética y visual. Salvo pequeñas excepciones, hallamos una realización sencilla con ciertos planos novedosos, y un ambiente sobrio y gris a pesar de contar con una alta iluminación. Además, en los dos productos los diálogos y la música constituyen factores esenciales, llegando a condicionar el significado de la escena; y el ritmo es especialmente ágil, con transiciones al corte y ausencia de fundidos.

Respecto a los elementos formales del relato, de forma genérica nos encontramos ante un modelo serial con subtramas episódicas que se presenta como dramedy. Aunque el número de episodios, temporadas y duración de los capítulos no es idéntico -OITNB continúa actualmente-, ambas obras incluyen una trama principal que se desarrolla a lo largo de la temporada, la cual no es totalmente resuelta al final de la misma, sino que sirve como gancho para la siguiente -recurso de narrativa audiovisual conocido como cliffhanger-; así como subtramas episódicas autoconclusivas. También en los dos casos hallamos una focalización externa, espacios interiores y exteriores, y una localización estadounidense.

En cuanto a los personajes, advertimos que configuran individuos difíciles y psicológicamente complejos, antihéroes que se alejan de los clichés y luchan por mejorar su situación actual dando lugar a un arco de personalidad cambiante. Además, en ambas series se incluyen personas de diferente raza, nacionalidad, religión, cultura y clase social; y se da un tratamiento muy específico a la mujer, ya sea por su protagonismo y poder en las tramas o por la supremacía numérica del género. En este sentido, Nancy Botwin y Piper Chapman responden a un mismo patrón, destacando su fuerte personalidad, ambigüedad sexual y necesidades emocionales.

En último lugar, a pesar de las acentuadas semejanzas narrativas y estéticas, las mayores similitudes se pueden advertir en la temática que desarrollan estas ficciones. Se trata de contenidos que se clasifican dentro de lo tabú: sexo, violencia, drogas, homosexualidad, ilegalidad... En este sentido, a pesar de que, como hemos anotado, dichos parámetros se podrían replicar en numerosas series de televisión contemporáneas, resulta de gran interés la simetría hallada en dos productos creados por el mismo profesional. En este caso, las ideas controvertidas se transmiten a través de mujeres aparentemente amables con las que el espectador se puede identificar fácilmente. La creadora consigue que el público empatice con personajes defectuosos para, a continuación, proponer una reflexión acerca de sus actitudes y las consecuencias de las mismas y, de este modo, alcanzar un nuevo significado que propicia un debate sobre la justificación de las acciones negativas. 
Se pone de manifiesto, por lo tanto, la relevancia e implicación de la figura del showrunner en el resultado final del proyecto. Kohan responde a este perfil profesional y desarrolla un estilo personal en sus series, el cual se hace patente no solo a través de los contenidos, pues también afecta y armoniza otros elementos de la narrativa y estética audiovisual, proporcionando la continuidad necesaria en un producto de esta tipología. En ambas ficciones hemos observado un conjunto de características comunes que constituyen la impronta creativa de su productora y determinan la huella que permite reconocer sus obras. De este modo, se pone en valor las funciones desempeñadas por el showrunner y su influencia sobre las cuestiones artísticas de la serie de ficción televisiva.

Para concluir, consideramos que el presente trabajo puede llegar a motivar la realización de futuros estudios similares que consideren el análisis de las aportaciones creativo-estilísticas de otros showrunners en series de televisión. De este modo, se fortalecerían o contrastarían los resultados hallados, y se ampliaría la investigación desarrollada.

\section{Referencias bibliográficas}

Ávila, F. (2013, 10 de Julio). Jenji Kohan es libre en la red. Excelsior. Recuperado de http://www.excelsior.com.mx/funcion/2013/07/10/908324

Barroso, J. (2002). Realización de los géneros televisivos. Madrid: Síntesis.

Bennett, T. (2014). Showrunners: The Art of Running a TV Show. London, England: Titan Books.

Buonanno, M. (2008). The Age of Television. Experiences and Theories. Bristol: Intellect.

Bordwell, D. y Thompson, K. (2012). Film Art, An Introduction (10 ${ }^{\text {th }}$ ed.). New York: McGraw-Hill.

Carrasco, A. (2010). Teleseries: géneros y formatos. Ensayo de definiciones. Miguel Hernández Communication Journal, 1(9), 174-200. Recuperado de http://rev.innovacionumh.es/index.php?journal $=$ mhcj\&page $=\operatorname{article} \& o p=v i e w \& p a t h \% 5 B \%$ $5 \mathrm{D}=22 \&$ path $\% 5 \mathrm{~B} \% 5 \mathrm{D}=42$

Cascajosa, C. (2005). Prime time: Las mejores series de TV americanas, de C.S.I. a Los Soprano. Madrid: Calamares Ediciones.

Cascajosa, C. (2006). No es televisión, es HBO: la búsqueda de la diferencia como indicador de calidad en los dramas del canal HBO. ZER Revista de Estudios de Comunicación, 11(21), 23-33. Recuperado de http://www.ehu.eus/zer/es/hemeroteca/articulo/No-es-televisin-es-HBO-La-bsqueda-de-la-diferencia-como-indicador-de-calidad-en-los-dramas-del-canal-HBO/291

Cascajosa, C. (2007). La caja lista: TV norteamericana de culto. Barcelona: Laertes. Cascajosa, C. (2016a). El ascenso de los "showrunners": Creación y prestigio crítico en la televisión contemporánea. Index Comunicación, 2(6), 23-40. Recuperado 
de http://journals.sfu.ca/indexcomunicacion/index.php/indexcomunicacion/article/ view/255/200

Cascajosa, C. (2016b). La cultura de las series. Barcelona: Laertes.

Clements, S. (2004). Showrunner. Producing variety and talk shows for television. Los Ángeles: Silman-James Press.

De Rosendo, N. y Gatell, J. (2015). Objetivo Writers'Room. Las aventuras de dos guionistas españoles en Hollywood. Barcelona: Alba.

Diego, P. (2005). The profile of the fiction producer in television. Communication \& Society 18(1), 9-30. Recuperado de http://www.unav.es/fcom/communicationsociety/es/articulo.php?art_id=63

Diego, P. (2010). La ficción en la pequeña pantalla. Navarra: EUNSA.

Douglas, P. (2011). Writing the TV Drama Series: How to Succeed as a Professional Writer in TV ( $3^{\text {rd }}$ ed.). Studio City, C.A.: Michael Wiese Productions.

Douglas, P. (2015). The future of television. Your Guide to Creating Television in the New World. Studio City, C.A.: Michael Wiese Productions.

Edgar Hunt, R., Marland, J. y Rawle, S. (2010). The Language of Film. Lausanne: AVA Publishing.

Fernández-Díez, F. y Martínez-Abadía, J. (1994). La dirección de producción para cine y $t v$. Barcelona: Paidós.

Fernández-Díez, F. y Martínez-Abadía, J. (2003). Manual básico de lenguaje y narrativa audiovisual. Barcelona: Paidós.

Fernández-Díez, F. y Martínez-Abadía, J. (2010). Manual del productor audiovisual. Barcelona: UOC.

Feuer, J. (1984). The MTM Style. En J. Feuer, P. Kerr y T. Vahimagi (Eds.), MTM: "Quality Television" (pp. 32-60). London, England: British Film Institute.

García de Castro, M. (2002). La ficción televisiva popular. Una evolución de las series de televisión en España. Barcelona: Gedisa.

García-Fanlo,L. (2016). El lenguaje de las series de televisión. Buenos Aires: EUDEBA.

Gervich, C. (2008). Small Screen, Big Picture. A Writer's Guide to the TV Business. New York: Three Rivers Press.

Gitlin, T. (2000). Inside prime time (4 ${ }^{\text {th }}$ ed.). Berkeley and Los Angeles: University of California Press.

Gómez-Martínez, P. J. (2015). La Dramedia: Rasgos diferenciadores y su proceso creativo. En B. Puebla-Martínez, N. Navarro-Sierra y E. Carrillo-Pascual (Eds.), Ficcionando en el Siglo XXI: La ficción televisiva en España (pp. 175-190). Madrid: Icono 14 Editorial.

Gordillo-Álvarez, I. (2009). Manual de narrativa audiovisual. Madrid: Síntesis.

Gross, T. y Miller, D. (2008, 16 de Junio). "Weeds" Creator Delivers Potent Product. Fresh Air. [Podcast]. Recuperado de http://www.npr.org/templates/story/story. php?storyId=91498188

Gross, T. y Miller, D. (2013, 13 de Agosto). "Orange” Creator Jenji Kohan: "Piper Was My Trojan Horse". Fresh Air. [Podcast]. Recuperado de http://www.npr.org/2013/08/13/211639989/ orange-creator-jenji-kohan-piper-was-my-trojan-horse

IMBd: Internet Movie Database (2017). Recuperado de http://www.imdb.com/ Jacoste, J. G. (2004). El productor cinematográfico (2a ed.). Madrid: Síntesis. 
Kallas, C. (2014). Inside the Writers' Room. Conversations with American TV Writers. Basingstoke: Palgrave Macmillan.

Kellison, C. (2009). Producing for TV and New Media: A Real-World Approach for Producers (2nd ed.). Burlington: Focal Press.

Kirkpatrick, S. (2015). Writing for the Green Light. How to Make your Script the One Hollywood Notices. New York: Focal Press.

Kubey, R. (2003). Creating Television. Conversations with the People Behind 50 Years of American TV. New York: Routledge.

Landau, N. (2014). The TV showrunner's roadmap. Burlington: Focal Press.

Longworth, J. L. (2000). TV creators: Conversations with America's top producers of television drama. New York: Syracuse UP.

Longworth, J. L. (2002). TV creators: Conversations with America's top producers of television drama, Vol. 2. New York: Syracuse UP.

Martin, B. (2013). Difficult Men: Behind the Scenes of a Creative Revolution: From The Sopranos and The Wire to Mad Men and Breaking Bad. New York: Penguin Group.

Millerson, G.(2009). Realización y producción en televisión (13 ${ }^{\mathrm{a}} \mathrm{ed}$.). Barcelona: Omega.

Nahum, A. (2014). El fenómeno de la serialidad en la Tercera Edad Dorada de la Televisión. En E. Fuster (Ed.), La figura del padre nella serialitá televisiva (pp. 19-42). Navarra: Universidad de Navarra.

Newcomb, H. y Alley, R. S. (1983). The Producer's Medium. Conversations with Creators of American TV. Oxford: Oxford University Press.

Newman, M. Z. y Levine, E. (2012). Legitimating Television: Media Convergence and Cultural Status. Nueva York: Routledge.

Pearson, R. (2005). The Writer/Producer, in American Television. En M. Hammond y L. Mazdon (Eds.), The Contemporary Television Series (pp. 11-26). Edinburgh: Edinburgh University Press.

Pérez-Gómez, M. A. (2011). Previously on. Intercisciplinary Studies on TV series in the third golden age of television. Sevilla: Biblioteca de la Facultad de Comunicación. Universidad de Sevilla.

Perren, A. (2011). In conversation: Creativity in the contemporary cable industry. Cinema Journal, 50(2), 132-138. Recuperado de https://works.bepress.com/ alisa_perren/9/

Perren, A. y Schatz, T. (2015). Theorizing television's Writer-Producer: Re-viewing The Producer's Medium. Television \& Media, 16(1), 86-93. Recuperado de http:// journals.sagepub.com/doi/10.1177/1527476414552907

Priggé, S. (2005). Created By... Inside the Minds of TV's Top Show Creators. Los Angeles: Silman-James Press.

Radish, C. (2013, 7 de Junio). Creator Jenji Kohan Talks "Orange is the New Black", Her Research Into Prison Life, and Graphic Sex Scenes. Collinder. Recuperado de http://collider.com/jenji-kohan-orange-is-the-new-black-interview/

Raya, I. (2012). El genre mixing en la ficción televisiva norteamericana. El caso de Joss Whedon. Revista de Comunicación, 1(10), 396-411. Recuperado de https:// idus.us.es/xmlui/handle/11441/31563

Sánchez-Noriega, J. L. (2006). El análisis del filme. En J. L. Sánchez-Noriega, Historia del cine: Teorías y géneros cinematográficos, fotografía y televisión (pp. 58-72). Madrid: Alianza Editorial. 
Sarris, A. (2004). Notes on the auteur theory in 1962. En G. Mast, M. Cohen y L. Braudy (Eds.), Film theory and criticism: Introductory readings (6th ed.) (pp. 561-564). Nueva York: Oxford University Press.

Schiff, R. y Holzman, W. (2014, 19 de Junio). Anatomy of a Script with Jenji Kohan: Part 1. Writers Guild Fundation. [Vídeo]. Recuperado de https://www.youtube. com/watch?v=EdJfmjc_jaY

Schiff, R. y Holzman, W. (2014, 19 de Junio). Anatomy of a Script with Jenji Kohan: Part 2.Writers Guild Fundation. [Vídeo]. Recuperado de https://www.youtube. com/watch?v=KzEC1n1X3yU

Steiner, T. (2015). Steering the Author Discourse: The Construction of Authorship in Quality TV, and the Case of Game of Thrones. International Journal of TV Serial Narratives, 1(2), 181-192. Recuperado de https://series.unibo.it/article/ view/5903

Thompson, E. y Mittell, J. (Eds.) (2013). How to Watch Television. New York: New York University Press.

Tous-Rovirosa, A. (2009). El concepto de autor en las series norteamericanas de calidad. En J. F. Serafim (Ed.), Autor e autoria no cinema e na televisão (pp.121169). Salvador: EDUFBA.

Tous-Rovirosa, A. (2010). La era del drama en televisión. Perdidos, CSI: Las Vegas, El ala oeste de la Casa Blanca, Mujeres desesperadas y House. Barcelona: Editorial UOCpress.

Valderrama, M., Boo, A. y Costas, N. (2013). Innovation and Transgression, Key to the Success of the Showtime Series. Revista Orbis, 26(9), 28-45. Recuperado de http://www.revistaorbis.org.ve/pdf/26/Art2.pdf

Willmore, A. (2013, 8 de Junio). "Weeds" Creator Jenji Kohan on Her New Netflix Series "Orange Is The New Black" and Why "Likability is Bullsh*t”. Indiewire. Recuperado de http://www.indiewire.com/article/ jenji-kohan-interview-orange-is-the-new-black

Wirth, J. y Melvoin, J. (2004). Writing for Episodic TV. From Freelance to Showrunner. Los Angeles: Writers Guild of America.

Zettl, H. (1996). El manual de producción para vídeo y televisión (6 ${ }^{\mathrm{a}} \mathrm{ed}$.). Andoain: Escuela de Cine y Televisión. 